\title{
Concurrent tACS-fMRI Reveals Causal Influence of Power Synchronized Neural Activity on Resting State fMRI Connectivity
}

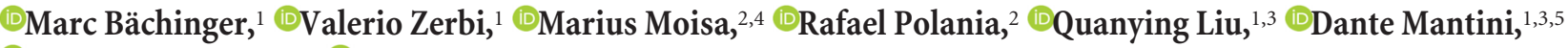 \\ (Christian Ruff, ${ }^{2,5}$ and ${ }^{-N i c o l e ~ W e n d e r o t h ~}{ }^{1,3,5}$ \\ ${ }^{1}$ Neural Control of Movement Laboratory, Department of Health Sciences and Technology, ETH Zürich, 8057 Zürich, Switzerland, ${ }^{2}$ Laboratory for Social \\ and Neural Systems Research, Department of Economics, University of Zürich, 8006 Zürich, Switzerland, ${ }^{3}$ Movement Control and Neuroplasticity Research \\ Group, Department of Kinesiology, KU Leuven, 3001 Leuven, Belgium, ${ }^{4}$ Institute for Biomedical Engineering, University and ETH of Zürich, 8052 Zürich, \\ Switzerland, and ${ }^{5}$ Neuroscience Centre Zürich, 8057 Zürich, Switzerland
}

Resting state fMRI (rs-fMRI) is commonly used to study the brain's intrinsic neural coupling, which reveals specific spatiotemporal patterns in the form of resting state networks (RSNs). It has been hypothesized that slow rs-fMRI oscillations $(<0.1 \mathrm{~Hz})$ are driven by underlying electrophysiological rhythms that typically occur at much faster timescales $(>5 \mathrm{~Hz})$; however, causal evidence for this relationship is currently lacking. Here we measured rs-fMRI in humans while applying transcranial alternating current stimulation (tACS) to entrain brain rhythms in left and right sensorimotor cortices. The two driving tACS signals were tailored to the individual's $\alpha$ rhythm $(8-12 \mathrm{~Hz})$ and fluctuated in amplitude according to a $1 \mathrm{~Hz}$ power envelope. We entrained the left versus right hemisphere in accordance to two different coupling modes where either $\alpha$ oscillations were synchronized between hemispheres (phase-synchronized tACS) or the slower oscillating power envelopes (power-synchronized tACS). Power-synchronized tACS significantly increased rs-fMRI connectivity within the stimulated RSN compared with phase-synchronized or no tACS. This effect outlasted the stimulation period and tended to be more effective in individuals who exhibited a naturally weak interhemispheric coupling. Using this novel approach, our data provide causal evidence that synchronized power fluctuations contribute to the formation of fMRI-based RSNs. Moreover, our findings demonstrate that the brain's intrinsic coupling at rest can be selectively modulated by choosing appropriate tACS signals, which could lead to new interventions for patients with altered rs-fMRI connectivity.

Key words: EEG; electrical stimulation; mechanism; neuronal oscillations; simultaneous tACS/fMRI

Significance Statement

Resting state fMRI (rs-fMRI) has become an important tool to estimate brain connectivity. However, relatively little is known about how slow hemodynamic oscillations measured with fMRI relate to electrophysiological processes. It was suggested that slowly fluctuating power envelopes of electrophysiological signals synchronize across brain areas and that the topography of this activity is spatially correlated to resting state networks derived from rs-fMRI. Here we take a novel approach to address this problem and establish a causal link between the power fluctuations of electrophysiological signals and rs-fMRI via a new neuromodulation paradigm, which exploits these power synchronization mechanisms. These novel mechanistic insights bridge different scientific domains and are of broad interest to researchers in the fields of Medical Imaging, Neuroscience, Physiology, and Psychology.

\section{Introduction}

Resting state fMRI (rs-fMRI) is a widely used tool for investigating large-scale functional connectivity within the human brain

\footnotetext{
Received June 1, 2016; revised March 30, 2017; accepted April 4, 2017.

Author contributions:M.B., M.M., R.P., D.M., C.R., and N.W. designed research;M.B.,M.M., and R.P. performed research; M.B., V.Z., Q.L., D.M., and N.W. analyzed data; M.B., V.Z., M.M., R.P., Q.L., D.M., C.R., and N.W. wrote the paper.

This work was supported by Swiss National Science Foundation 320030_149561 and 320030_146531, the National Center for Competence in Research Affective Sciences, FW0 Flanders G.0401.12, and Seventh Framework Programme European Commission PCIG12-2012-334039. We thank Karl Treiber for scanning assistance; and Daniel Woolley for feedback on the manuscript.

The authors declare no competing financial interests.
}

(Biswal et al., 1995; Fox and Raichle, 2007). rs-fMRI measures spontaneous slow fluctuations (i.e., $<0.1 \mathrm{~Hz}$ ) of the BOLD signal at rest. These fluctuations form spatial patterns of correlated activity that can be mapped onto resting state networks (RSNs); these exhibit a unique topography that often resembles networks activated by specific tasks (i.e., sensorimotor network, visual

Correspondence should be addressed to Marc Bächinger, Neural Control of Movement Laboratory, Department of Health Sciences and Technology, ETH Zürich, Winterthurerstrasse 190, 8057 Zürich, Switzerland. E-mail: marc.baechinger@hest.ethz.ch.

DOI:10.1523/JNEUROSCI.1756-16.2017

Copyright $\odot 2017$ the authors $\quad 0270-6474 / 17 / 374766-12 \$ 15.00 / 0$ 
network, etc.) (Damoiseaux et al., 2006). Alterations in restingstate connectivity have been associated with several neuropathologies (Greicius, 2008; Bullmore and Sporns, 2009; Fornito and Bullmore, 2010; Alaerts et al., 2014, 2016) opening new opportunities for identifying disease-specific biomarkers and potential therapy targets at the brain circuit level (Alaerts et al., 2014; Woolley et al., 2015). However, which exact electrophysiological mechanisms cause RSNs to emerge is still debated, making it difficult to design new interventions that target activity at the cell population level with the aim of normalizing large-scale connectivity within specific circuits. Previous studies have linked rsfMRI connectivity to ultra-slow $(<0.5 \mathrm{~Hz})$ fluctuations of electrophysiological signals ( $\mathrm{Pan}$ et al., 2013), or to positively correlated power envelopes (also called band-limited power signals) of faster frequency bands, including the $\delta(<4 \mathrm{~Hz})$ (Lu et al., 2007), $\alpha(8-12 \mathrm{~Hz}) / \beta(12-30 \mathrm{~Hz}$ ) (Mantini et al., 2007; Brookes et al., 2011; Wang et al., 2012) and $\gamma$ bands (He et al., 2008; Nir et al., 2008; Schölvinck et al., 2010). This converging evidence indicates that slowly fluctuating components of the electrophysiological signal (either the power envelope or the signal itself) synchronize across different areas of the brain, and the topography of this synchronization pattern has been linked to heightened BOLD connectivity within corresponding RSNs.

However, these previous studies revealed mainly correlative evidence arguing that the topography or strength of connectivity is similar when RSNs determined from synchronized activity of the BOLD signal were compared with RSNs determined from electrophysiological readouts (LFP or EEG) (Lu et al., 2007; He et al., 2008; Nir et al., 2008; Brookes et al., 2011; Wang et al., 2012; Hipp and Siegel, 2015). Here we used a novel interventional approach to experimentally probe the hypothesis that synchronizing power envelopes of the prominent $\alpha$ rhythm across anatomically connected brain areas increases rs-fMRI connectivity within the targeted network. The basic idea is to modulate electrophysiological activity with noninvasive electrical stimulation applying appropriately designed, EEG-based driving signals while measuring the induced changes in rs-fMRI connectivity. This approach is not only interesting as a research tool but also as a potential intervention for modulating large-scale connectivity at rest within clinically relevant circuits.

\section{Materials and Methods}

We applied transcranial alternating current stimulation (tACS) to entrain endogenous neuronal oscillations in a frequency-dependent way (Zaehle et al., 2010; Polanía et al., 2012; Herrmann et al., 2013; Helfrich et al., 2014; Cecere et al., 2015) without causing significant interference with MRI measurements (Antal et al., 2014). Using a 3-electrode setup to drive neural activity within left and right sensorimotor cortices (SM1), functional coupling between hemispheres can be either strengthened or weakened by administering two iso-frequent stimulation signals, which are either in-phase or antiphase, respectively (Polanía et al., 2012, 2015). The waveforms were tailored to the subject's individual $\alpha$ frequency band (IAF; corresponding to $\sim 8-12 \mathrm{~Hz}$ oscillation), which was chosen as a "carrier frequency" because it is a strong endogenous rhythm present at rest. Moreover, in motor cortex, the power envelope of the $\alpha$ band has been linked to interhemispheric connectivity measured by EEG/MEG (Siems et al., 2016), rs-fMRI connectivity (Mantini et al., 2007), and task-based fMRI activity (Ritter et al., 2009). Importantly, we modulated the amplitude of the tACS signals according to a $1 \mathrm{~Hz}$ envelope, thereby mimicking a cross-frequency coupling phenomenon (i.e., a phaseamplitude coupling mechanism between the $\alpha$ and $\delta$ rhythm) that emerges in humans at rest (Siems et al., 2016). We then aimed to disambiguate whether interhemispheric rs-fMRI connectivity is mainly driven by the interhemispheric synchronization of $1 \mathrm{~Hz}$ amplitude envelopes or by the synchronization of left and right IAF. We therefore stimulated
SM1 of each hemisphere with the same amplitude-modulated IAF driving signal, but with different phase relationships between hemispheres. In the power-synchronized tACS condition, the $1 \mathrm{~Hz}$ amplitude envelopes were in-phase, but the IAF signals were antiphase; by contrast, in the phase-synchronized tACS condition, the IAF signals were in-phase, but the $1 \mathrm{~Hz}$ amplitude envelopes were antiphase. Importantly, the power-synchronized tACS condition approximates the interhemispheric coupling as measured beforehand with EEG at rest, whereas the phasesynchronized tACS inverts this interhemispheric phase relationship and acts as negative control. We hypothesized that the power-synchronized stimulation regimen would increase rs-fMRI connectivity between hemispheres.

Experimental design. The experiment consisted of two sessions, which were on average 1 week apart. During the first session, 5 min of restingstate EEG (eyes-open) was measured and the participants' EEG frequency spectrum was prescreened. Only subjects with a clear $\alpha$ peak participated in the tACS/fMRI experiment. Based on the prescreening, we tailored the tACS stimulation signals to the IAF. In the second session, a combined tACS/rs-fMRI (eyes-open) experiment was conducted to test the effects of two different stimulation paradigms on rs-fMRI connectivity between the sensorimotor cortices of the two hemispheres.

Subjects. Thirty-five subjects were recruited for the EEG session. All subjects provided informed consent as approved by the Research Ethics Committee of the Canton of Zurich. Subjects were prescreened to have a clear $\alpha$ peak over sensorimotor areas (at electrode C3 or C4; for definition of $\alpha$ peak, see EEG analysis). Twenty-two subjects showed an $\alpha$ peak over both hemispheres. Their EEG was analyzed in more detail, and they participated in the subsequent tACS/fMRI experiment. From the 22 subjects who were scanned, two were excluded from analysis: one because of too much movement and one fell asleep during the experiment. Twenty subjects were analyzed, and all reported results (EEG and fMRI) are from those subjects unless stated otherwise $(n=20$, age $=24.8 \pm 4.1$ years, mean \pm SD, 10 females, 19 right-handed by self-indication).

EEG acquisition. EEG was measured using a 128-channel HydroCel Geodesic Sensor Net using $\mathrm{Ag} / \mathrm{AgCl}$ electrodes provided by Electrical Geodesics. This system uses the vertex $(\mathrm{Cz})$ electrode as physical reference. EEG recordings, electro-oculograms for horizontal and vertical eye movements, respectively, and an electromyogram for the muscular noise associated with swallowing were recorded in parallel with a sampling frequency of $1000 \mathrm{~Hz}$.

During EEG acquisition, subjects sat in a dark room and fixated on a cross presented on a computer screen in front of them for $5 \mathrm{~min}$. The unfiltered data were saved for offline analyses.

EEG preprocessing. EEG signals were bandpass filtered off-line (2-40 $\mathrm{Hz}$ ) and further processed using independent component analysis (ICA) for the removal of ocular and muscular artifacts with eeglab (Delorme and Makeig, 2004). After ICA decomposition, the artifact ICs were automatically detected by correlating their power time courses with the power time courses of the electric reference signals: the horizontal electrooculogram, the vertical electro-oculogram, and electromyogram at the base of the neck. The data were then downsampled to $200 \mathrm{~Hz}$ and rereferenced to the common average (Liu et al., 2015) to remove the bias toward the physical reference site (Luck, 2014).

EEG source localization. After the fMRI experiment (see below), source localization of the EEG data was performed to control that the results obtained in sensor space indeed reflected activity in sensorimotor areas. A forward head model was built with the finite element method, using a 12-tissue head template and the standard electrode positions for a 128channel EGI cap. The head template was obtained from the IT'IS foundation of ETH Zurich (Iacono et al., 2015) and included 12-tissue classes (skin, eyes, muscle, fat, spongy bone, compact bone, cortical gray matter, cerebellar gray matter, cortical white matter, cerebellar white matter, CSF, and brainstem). Specific conductivity values were associated with each tissue class (i.e., skin $04348 \mathrm{~S} / \mathrm{m}$, compact bone $0.0063 \mathrm{~S} / \mathrm{m}$, spongy bone $0.0400 \mathrm{~S} / \mathrm{m}$, CSF $1.5385 \mathrm{~S} / \mathrm{m}$, cortical gray matter $0.3333 \mathrm{~S} / \mathrm{m}$, cerebellar gray matter $0.2564 \mathrm{~S} / \mathrm{m}$, cortical white matter $0.1429 \mathrm{~S} / \mathrm{m}$, cerebellar white matter $0.1099 \mathrm{~S} / \mathrm{m}$, brainstem $0.1538 \mathrm{~S} / \mathrm{m}$, eyes $0.5000 \mathrm{~S} / \mathrm{m}$, muscle $0.1000 \mathrm{~S} / \mathrm{m}$, and fat $0.0400 \mathrm{~S} / \mathrm{m}$ ) (Haueisen et al., 1997). The dipoles corresponding to brain sources were placed on a regular $6 \mathrm{~mm}$ 
grid spanning cortical and cerebellar gray matter. After the head model template was established, the brain activity in each dipole source was estimated by the exact low-resolution brain electromagnetic tomography (eLORETA) (Pascual-Marqui et al., 2011) for each subject.

EEG analysis. EEG analysis was performed using the fieldtrip toolbox (Oostenveld et al., 2011) and custom Matlab (The MathWorks) scripts. Group analyses in sensor space were verified by parallel analyses in source space, whereas individual-difference analyses were restricted to sensor space to minimize confounds caused by imperfect source localization. For each subject, the individual $\alpha$ peak and the correlational properties of the $\alpha$ rhythm during rest were determined for electrodes C3 and C4 (sensor space), which corresponds to the left and right primary sensorimotor cortices, respectively.

For determining the individual $\alpha$ peak, we first calculated the frequency spectrum for each electrode using the absolute of the Fourier transform. The individual $\alpha$ peak was determined as the local maximum between 7 and $14 \mathrm{~Hz}$ of the resulting spectrum. Only subjects with a clearly identifiable $\alpha$ peak in sensor space when visually inspected were included in the fMRI experiment. The IAF band for each subject was then defined as individual $\alpha$ peak $\pm 2 \mathrm{~Hz}$ (Zaehle et al., 2010). We then bandpass filtered the signal within the IAF of each subject (IAF signal). We also calculated the amplitude envelopes of the IAF signals using the absolute of the Hilbert transform. Next, the natural phase relationship between the two IAF signals and the two amplitude envelopes of $\mathrm{C} 3$ and C4 was determined via the Pearson's correlation coefficient $r$ and via the instantaneous phase relationship. The latter was calculated by epoching the resting-state EEG data into segments of $5 \mathrm{~s}$. For each segment, we calculated the frequency spectrum, the instantaneous phase of the IAF signal, and the instantaneous phase of the amplitude envelope. We averaged the frequency spectrum across all $5 \mathrm{~s}$ segments and determined mean IAF peaks and 95\% CIs. The plots were visually inspected for each individual to confirm that the IAF exhibited a clear peak in the $5 \mathrm{~s}$ intervals to ensure that the relative phase would represent meaningful values (see Fig. 1A). Instantaneous phase difference of the IAF signal and the amplitude envelope between C3 and C4 was calculated for each subject, and the main directionality of the phase difference across subjects was statistically assessed using $\mathrm{V}$ tests for circular uniformity. In short, V tests assess whether a vector of angles has a known main direction (Berens, 2009). We tested $180^{\circ}$ for the IAF signal and $0^{\circ}$ for the amplitude envelopes. The same phase analysis was then performed in source space: for the first principal component of the signals extracted from the center of gravity $(\operatorname{cog})$ of the left and right part of the sensorimotor network as identified during the fMRI analysis (cog left hemisphere: MNI - 15, -29, $63, \operatorname{cog}$ right hemisphere: MNI $13,-28,63)$. This was done to confirm that the signals measured in $\mathrm{C} 3 / \mathrm{C} 4$ originated from the sensorimotor network.

tACS 3-electrode setup. We used two modified MR compatible DC stimulator plus devices (Neuroconn) that were connected to two active electrodes and one common return electrode (electrode-size $5 \times 7 \mathrm{~cm}$ ). The active electrodes were placed over the left and right motor cortex as determined by transcranial magnetic stimulation (hotspot of the first dorsal interosseus muscle) and the return electrode was placed $\sim 2-3 \mathrm{~cm}$ above the inion to minimize the perception of phosphenes while preventing that subjects would not lie on the return electrode while scanning (which might have caused discomfort).

The stimulation was controlled via the REMOTE connectors of the stimulators which allows externally generated voltage signals to be translated into current/stimulation signals. The actual stimulation signals were produced with a custom-made MATLAB script and sent to the stimulators via a National Instruments Card (NI-USB 6343). This allowed precise control of the output signals (especially phase stability between the two signals).

The stimulation signals were individualized to the subject's IAF. If the individual peak frequency differed between left and right hemispheres, then the average of the two was used. Importantly, because the precise frequency of the individual $\alpha$ peak might change slightly from the EEG session to the fMRI session (compare Vossen et al., 2015), we used stimulation signals that covered the whole IAF band ( $\alpha$ peak $\pm 2 \mathrm{~Hz}$ ). The signals were based on the subject's IAF that was modulated with a $1 \mathrm{~Hz}$ envelope (see Fig. 1D). The $1 \mathrm{~Hz}$ envelope was chosen based on pilot data where the group average frequency spectrum had a broad peak from 0.5 to $2 \mathrm{~Hz}$ with a maximum at $1 \mathrm{~Hz}$. We used this value for designing the tACS waveforms used in the main experiment. We repeated this analysis with the full sample, which revealed a group maximum at $1.2 \mathrm{~Hz}$. All these analyses were performed after applying a bandpass filter to the EEG signal with cutoffs at the IAF $\pm 2 \mathrm{~Hz}$. The maximum current intensity (peak-to-peak amplitude) over the active electrode was $1.5 \mathrm{~mA}$ and 0.75 $\mathrm{mA}$ during the peak and trough of the envelope, respectively.

Two different phase relationships between the left and right hemisphere were tested for the experiment. During power-synchronized tACS, the two stimulation signals were in antiphase $\left(180^{\circ}\right.$ degrees phaseshifted) and the envelopes were in-phase ( $0^{\circ}$ phase shifted). During phase-synchronized tACS, the stimulation signals were in-phase $\left(0^{\circ}\right.$ phase shifted) and the envelopes were antiphase ( $180^{\circ}$ phase-shifted). Power-synchronized tACS represents a synchronization of the power envelopes similar to that observed for the EEG signals measured over C3 and C4, whereas phase-synchronized tACS inverts this phase relationship (i.e., no synchronization of the power envelope) and is used as negative control condition.

MRI acquisition. Resting-state imaging was performed at the Laboratory for Social and Neural Systems research of the University of Zurich, on a Philips Achieva 3T whole-body scanner equipped with an eightchannel MR head coil. Before applying tACS during fMRI, we did basic safety and quality tests for the 3-electrode setup. We tested for dynamic tACS artifacts and for heating under the tACS electrodes, following the protocol described previously (Moisa et al., 2016). In short, the conducted analyses did not reveal dynamic artifacts due to our tACS stimulation or an increase in temperature due to up to $30 \mathrm{~min}$ of stimulation. Moreover, data-driven artifact removal performed with FSL-FIX (see rs-fMRI analysis) did not reveal any components with a topography that would suggest tACS-related artifacts (e.g., we did not see components under/close to the electrodes).

Initially, T1-weighted 3D turbo field echo B0 scans were acquired for correction of possible static distortion produced by the presence of the active electrode (voxel size $=3 \times 3 \times 3 \mathrm{~mm}^{3}, 0.5 \mathrm{~mm}$ gap, matrix size $=$ $80 \times 80$, TR/TE $1 /$ TE2 $=418 / 4.3 / 7.4 \mathrm{~ms}$, flip angle $=44$, no parallel imaging, 37 slices). High-resolution T1-weighted 3D turbo field echo structural scans were acquired and used for image registration and normalization (181 sagittal slices, matrix size $=256 \times 256$, voxel size $=1$ $\mathrm{mm}^{3}$, TR/TE/TI $\left.=8.3 / 2.26 / 181 \mathrm{~ms}\right)$. Thereafter, five resting-state runs of 7 min were collected for each subject (2-3 min break between each block). Each resting-state run contains 200 volumes (voxel size $=3 \times$ $3 \times 3 \mathrm{~mm}^{3}, 0.5 \mathrm{~mm}$ gap, matrix size $=80 \times 80, \mathrm{TR} / \mathrm{TE}=2100 / 30 \mathrm{~ms}$, flip angle $=79$, parallel imaging factor $=1.5,35$ slices acquired in ascending order for full coverage of the brain).

The first run served as a baseline measurement. During the second and fourth runs, tACS was applied (the order of tACS conditions was counterbalanced between subjects). Runs 3 and 5 captured potential aftereffects (see Fig. 1). This allowed us to compare the effect of each stimulation protocol on rs-fMRI connectivity in relation to baseline, but also to directly contrast the effects of power-synchronized versus phasesynchronized tACS stimulation.

rs-fMRI analysis. fMRI analysis was performed similar to the protocol previously used by Stagg et al. (2014). Brain functional networks (i.e., independent components) were identified using the Multivariate Exploratory Linear Optimized Decomposition into Independent Components (MELODIC; version 3.10) module in FSL (fMRIB's Software Library, www.fmrib.ox.ac.uk/fsl). Standard preprocessing consisted of motion correction, brain extraction, spatial smoothing using a Gaussian kernel of FWHM $8.0 \mathrm{~mm}$, and high-pass temporal filtering (100 s/0.01 $\mathrm{Hz}$ ). Additionally, artifact components from non-neural sources were removed with FSLs fMRIB's ICA-based Xnoiseifier FSL-FIX (Griffanti et al., 2014; Salimi-Khorshidi et al., 2014). In short FSL-FIX automatically classifies each component into either signal or noise and regresses the noise components from the original data using the standard classifier at threshold 20. In comparison with a manual classification done by two experts on a subset of the subjects $(n=5)$, FSL-FIX yielded a very high sensitivity (97.6\%) in detecting true RSNs. 

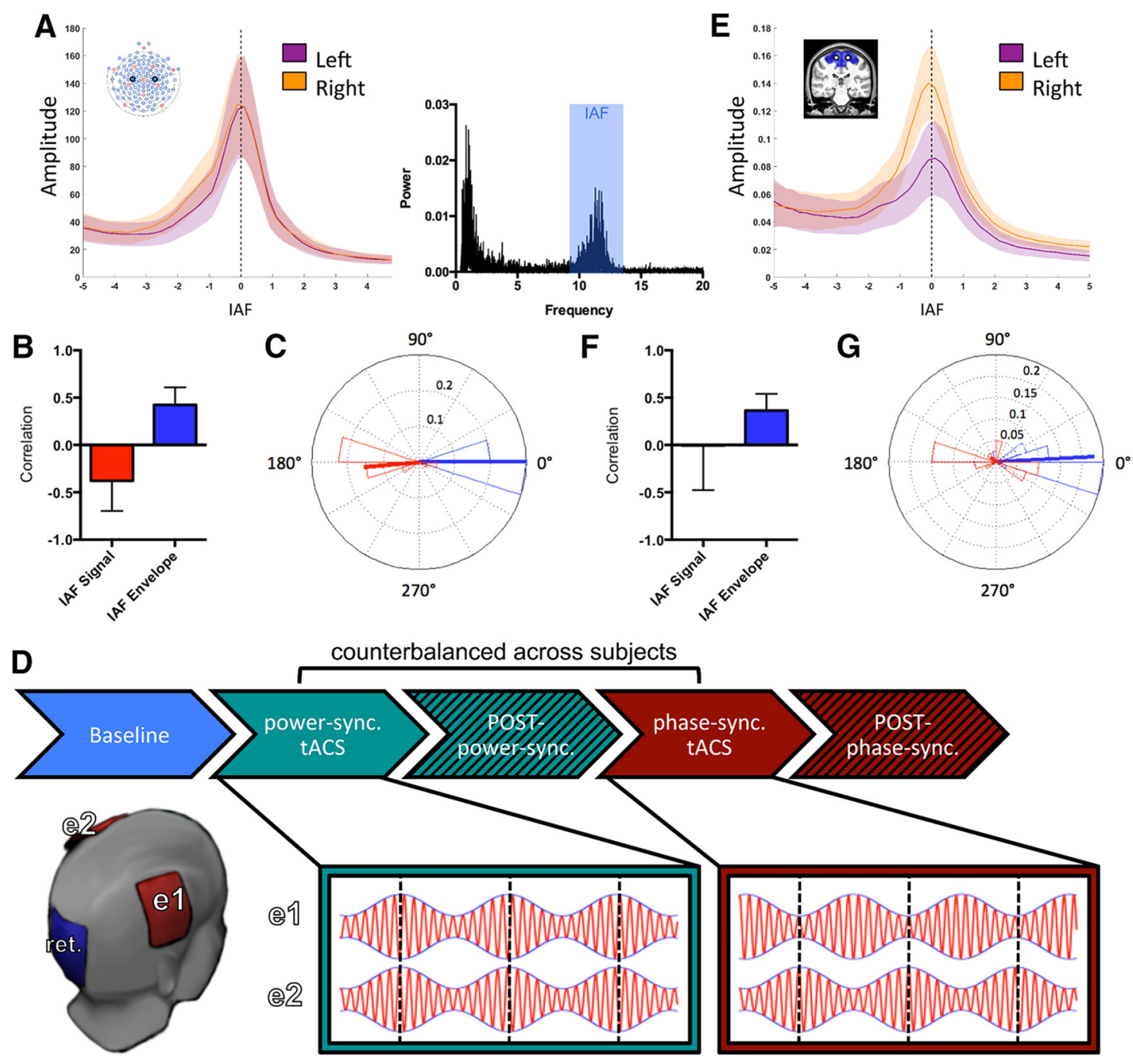

Figure 1. EEG-based stimulation signals during fMRI. $\boldsymbol{A}$, For each subject, the individual $\alpha$ peak and IAF band (IAF $=\alpha$ peak $\pm 2 \mathrm{~Hz}$ ) was determined from electrodes $(3 / \mathrm{C} 4$ during rest (eyes-open). Right, Exemplary frequency spectrum. Left, Mean and $95 \% \mathrm{CI}$ (shaded area) of the EEG frequency spectrum normalized to the IAF $\pm 5 \mathrm{~Hz}$ of the left and the right hemisphere, indicating a clear peak. B, Correlational analysis showing an anticorrelated relationship between electrodes $C 3 / C 4$ for the signal within IAF (red) and a positive correlation for the envelope modulation of IAF (blue). C, Histogram of relative phase difference between $(3$ and C 4 of IAF signal (red) and envelope (blue) across subjects. Arrows indicate average phase difference across subjects. D, Based on the subject's IAF, two kinds of stimulation signals were applied via a 3-electrode setup during rs-fMRI. Power-synchronized tACS mimics the in-phase relationship of the IAF power envelopes between C3/C4 (while the IAF signal was anticorrelated; blue-green) and phase-synchronized tACS (control) with the opposite correlational properties (antiphase power fluctuations, synchronized IAF signal). A total of 35 min of resting state fMRI was recorded, split into 5 runs of 7 min from the same 20 subjects as in the EEG experiment (see Materials and Methods). During runs 2 and 4 , powersynchronized and phase-synchronized $t A C S$ was applied via 2 active electrodes placed on the left and right motor cortex (red); run 1 served as baseline for further analysis, and runs 3 and 5 measured potential aftereffects. $E$, Post-fMRI analysis of the EEG data confirms that the IAF is clearly detectable in source space (i.e., in left and right sensorimotor cortex). $F, G$, Correlational and instantaneous phase properties in source space. The IAF envelope exhibits robust synchronization (i.e., O degrees relative phase) between hemispheres, whereas the IAF signals exhibit large intersubject variability.

The artifact-cleaned functional data were then aligned to structural images and normalized into MNI space using linear and nonlinear transformations (advanced normalization tools) (http://stnava.github.io/ANTs).

Normalized functional data for each subject were temporally concatenated across subjects to create a single 4D dataset, and group ICA was performed to identify RSNs. Between-subject analysis was performed using a dual regression approach implemented in FSL (Beckmann et al., 2009). In short, this approach consists of two stages: (1) a spatial regression of the data is calculated to identify the time course of a RSN; and (2) a temporal regression with those time courses is determined to get the subject specific map of the RSN. This is done for each RSN.

The resulting subject-specific component map was then masked by the 75th percentile group mean RSN map. The mean value of the parameter estimates within this region was extracted for each subject. The average of this parameter estimate can be seen as a measure of the average strength of functional connectivity within each RSN. This analysis was performed for each RSN separately.
The average scores of each subject were then submitted to a linear mixed-effects model (LMEM) with the fixed factor stimulation type (5 levels, repeated measure) and the random factor subject. Corresponding contrasts were used for post hoc pairwise comparisons (LSD) for which effect sizes were calculated using Cohen's $d$ as an intuitive estimate. This was done for each RSN of interest separately.

Finally, a qualitative voxelwise analysis was performed for each RSN using nonparametric paired $t$ tests (threshold free cluster enhancement; tfce corrected) as implemented by randomize in FSL (Winkler et al., 2014).

Modeling of the electric field induced by tACS. We compared the electric field induced by phase-synchronized versus power-synchronized tACS using SimNIBS 2.0 (Thielscher et al., 2015) to model the effect. A realistic finite element head model was used for the simulations. We simulated the same electrode placement as during the experiment (i.e., above the left and right hand knob region in M1 and the return electrode $2 \mathrm{~cm}$ above the inion). The stimulation signals were downsampled to $80 \mathrm{~Hz}$, 
A

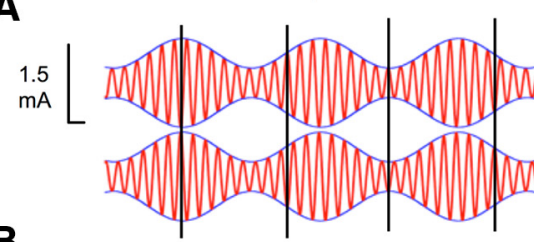

Left Hemisphere
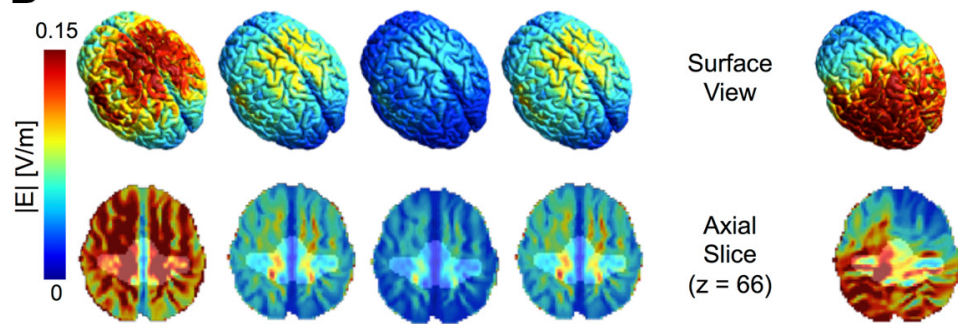

Axial

Slice

$(z=66)$

Phase-sync. tACS

C

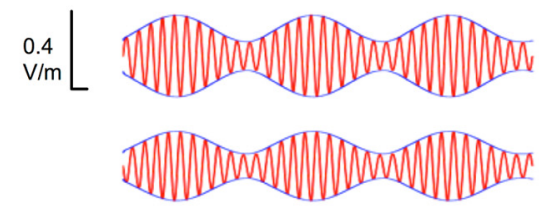

Simulated Signals

$$
\begin{gathered}
\begin{array}{c}
\text { Sensorimotor System } \\
\text { (Left) }
\end{array} \\
\text { (MNI -18 40 66) } \\
\text { Sensorimotor System } \\
\text { (Right) } \\
\text { (MNI } 184066)
\end{gathered}
$$

Visual System (MNI 2 -88 22)
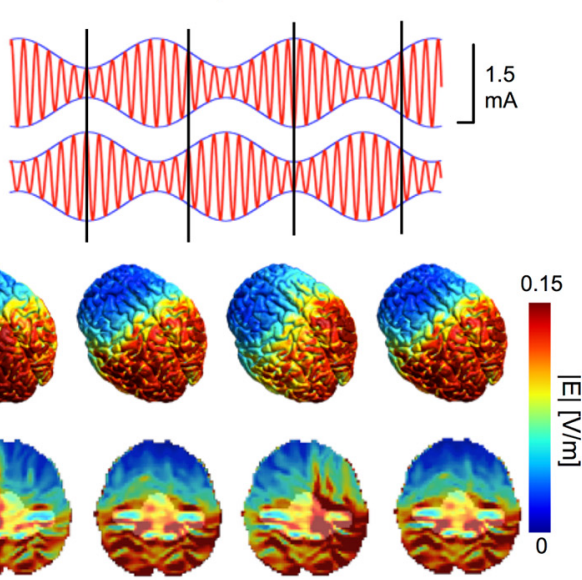

Simulated Signals
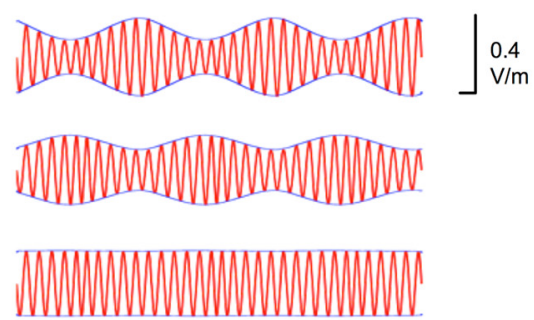

Figure 2. Simulations of the electric field during power-synchronized (left side) and phase-synchronized tACS (right side). $\boldsymbol{A}$, Top, Externally applied signals over left and right sensorimotor cortex. $\boldsymbol{B}$, The electric field distribution estimated for four different time points of the envelope amplitude of the right hemisphere (indicted by black vertical lines) is shown on a surface view and an axial slice through the sensorimotor system $(z=66)$. The highlighted aspect shows the sensorimotor RSN. C, Simulated signals showing effective modulation within left and right sensorimotor cortex extracted from MNI coordinates $+/-18,-40,66$ (i.e., the maximum of the simulated electric field during power-synchronized stimulation) and the visual cortex (MNI 2, $-88,22$; i.e., approximately under the return electrode). During power-synchronized tACS $(B, C$, left), both hemispheres oscillate synchronously between strong and weak stimulation. By contrast, during phase-synchronized tACS $(\boldsymbol{B}, \boldsymbol{C}$, right), one hemisphere is always more strongly activated than the other. Also, the maximum modulation depth of the envelope reached similar levels in the sensorimotor cortex for both stimulation conditions $(0.25 \mathrm{~V} / \mathrm{m}$ for power-synchronized tACS and $0.21 \mathrm{~V} / \mathrm{m}$ for phase-synchronized tACS).

and a whole brain image of the electric field distribution was calculated for each time point. For visualization purposes, we extracted the norm of the electric field during peaks and troughs of the envelopes of powersynchronized and phase-synchronized stimulation. Furthermore, we investigated how the field fluctuated within our target area: the sensorimotor network by reconstructing the signal within a $4 \mathrm{~mm}$ circular seed region at the peak of the electric field during power-synchronized stimulation (MNI: $-18,-40,66$ ). This was done by taking the first principal component of the electric field and upsampling the signal to the original sampling rate of $1280 \mathrm{~Hz}$ for visualization purposes (see Fig. 2).

\section{Results}

\section{Designing EEG-based tACS signals}

We recorded and analyzed resting-state EEG in 20 subjects and identified their IAF band (IAF, $2 \mathrm{~Hz}$ below and above the individual $\alpha$ peak; for inclusion criteria, see Materials and Methods) over left and right SM1 (sensor space, electrodes C3 and C4, respectively; Fig. 1A) (Zaehle et al., 2010).

The IAF signals (peak $\alpha$ frequency was $10.6 \pm 1.2 \mathrm{~Hz}$ with an average amplitude of $0.22 \pm 0.11 \mu \mathrm{V}$ over the left hemisphere and $10.5 \pm 1.1 \mathrm{~Hz}$ with an average amplitude of $0.21 \pm 0.13 \mu \mathrm{V}$ over right hemisphere; all values mean $\pm \mathrm{SD}$ ) measured in $\mathrm{C} 3 / \mathrm{C} 4$ were on average negatively correlated $\left(\mathrm{r}_{\mathrm{IAF}}=-0.38 \pm 0.32\right)$ and exhibited an instantaneous relative phase $\Phi_{\mathrm{IAF}}=-174.8 \pm 45.2^{\circ}$, indicating that the IAF signals of the left and right hemisphere oscillated in antiphase (Fig. 1C, red symbols). Next, we characterized the IAF envelope (within a $0.1-2 \mathrm{~Hz}$ band), which was positively correlated between hemispheres $\left(\mathrm{r}_{\mathrm{Env}}=0.42 \pm 0.19\right)$ and tended to oscillate in-phase $\left(\Phi_{\text {Env }}=0.02 \pm 0.21^{\circ}\right.$, Fig. $1 B, C$, blue symbols). Using the sensorimotor network from the fMRI analysis (see below), we checked whether the correlational and instantaneous phase properties were still present in source space (Fig. $1 E-G$ ). The peak $\alpha$ frequency was comparable with the values obtained in sensor space (left hemisphere: $9.6 \pm 0.9 \mathrm{~Hz}$, right hemisphere: $9.7 \pm 0.9)$. The correlational and instantaneous phase differences of the envelopes were also comparable with the sensor data $\left(\mathrm{r}_{\text {Env,Source }}=0.37 \pm 0.19, \Phi_{\text {Env, Source }}=3.2 \pm 23.9^{\circ}\right)$. The phase offset of the IAF signal in source space trended toward antiphase; however, intersubject variability was much larger than in sensor space $\left(\Phi_{\mathrm{IAF}}=-138.6 \pm 76.6^{\circ}\right)$ such that the signals were uncorrelated when averaged across subjects $\left(\mathrm{r}_{\mathrm{IAF} \text {,Source }}=\right.$ $0.00 \pm 0.48$ ). This further emphasizes that the low-frequency envelope, rather than the IAF, signals exhibit synchronous oscillations between hemispheres.

We then designed the tACS waveforms with the IAF of each subject as the main carrier frequency, while the amplitude was modulated with a fluctuating $1 \mathrm{~Hz}$ envelope (maximal intensity $=1.5 \mathrm{~mA}$ peak-to peak, amplitude modulation $=0.5$ of maximal intensity). We used two stimulation regimens: In the phase-synchronized condition, the relative phase of IAF was $0^{\circ}$ $\left(\Phi_{\mathrm{IAF}}=0^{\circ}\right)$ and the relative phase of the $1 \mathrm{~Hz}$ envelope was $180^{\circ}$ $\left(\Phi_{\text {Env }}=180^{\circ}\right)$, whereas this was reversed in the powersynchronized condition $\left(\Phi_{\mathrm{Env}}=0^{\circ}\right.$ and $\Phi_{\mathrm{IAF}}=180^{\circ}$; Fig. $\left.1 D\right)$. Power-synchronized tACS approximates the interhemispheric coupling measured with EEG at rest (correlated envelopes, anticorrelated/uncorrelated IAF signals). Next, we modeled the 


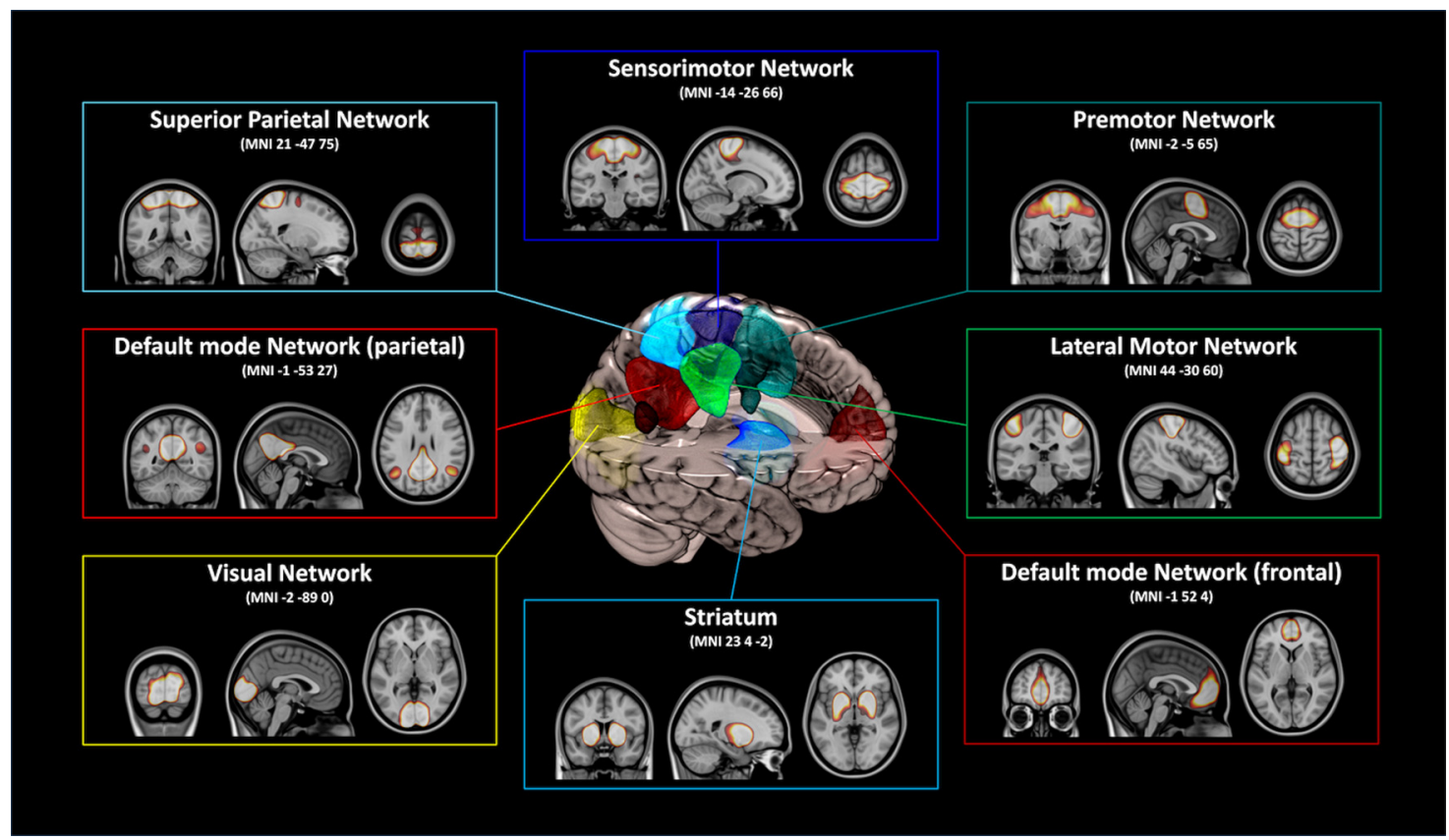

Figure 3. RSNs of interest. Based on the MELODIC group-ICA, eight RSNs were selected for further analyses: a sensorimotor network (blue), a premotor network (blue-green), a more lateral motor network (light green), the default-mode network split into a frontal (dark red) and parietal part (light red), the striatum (light blue, bottom), a visual network (yellow), and a superior parietal network (light blue, top).

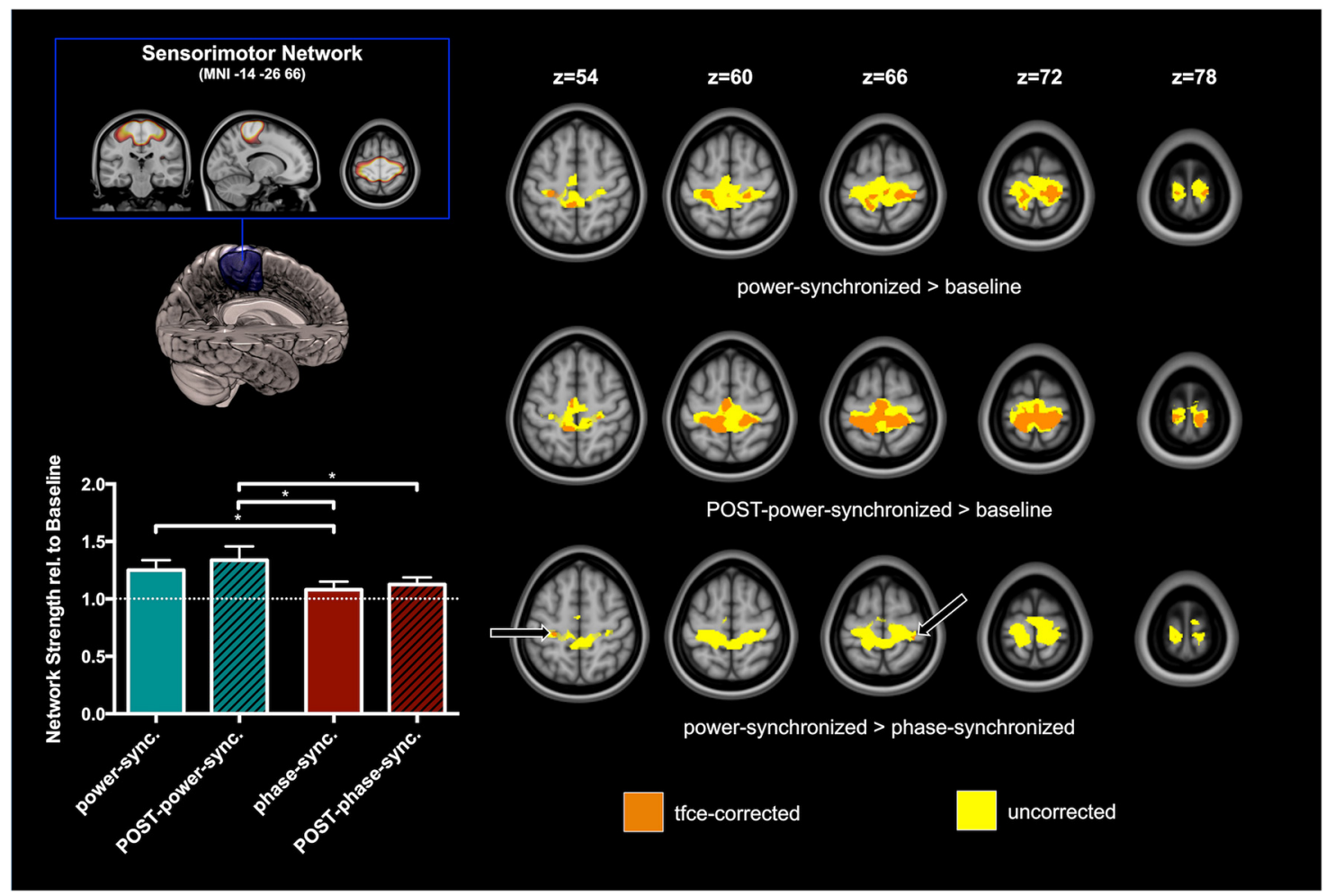

Figure 4. Increase of network strength of the sensorimotor network during power-synchronized tACS compared with baseline and phase-synchronized tACS (control). Left, Averaged normalized scores of network strength identified by dual regression during and after power-synchronized (blue-green) and phase-synchronized tACS (dark red) revealing an increase of $25 \%$ in network strength during power-synchronized tACS compared with baseline and a significant increase (20\%; $p=0.037)$ compared with phase-synchronized tACS (control). The increase was still significant when comparing the two aftereffects (POST power-synchronized and POST phase-synchronized, $p=0.010$ ). ${ }^{*} p<0.05$. Error bars indicate mean \pm SEM. Right, Corresponding voxelwise contrasts revealing an increase of network strength around the central sulcus in between the active electrodes during and after power-synchronized tACS compared with baseline and phase-synchronized tACS, and a general increase during and after power-synchronized tACS compared with baseline (all images: $\mathrm{p}_{\text {tfce-corrected }}<0.05$ ).

electric field to assess whether our two stimulation paradigms caused comparable effects in sensorimotor cortex. We found that both the amplitude of the $\alpha$ oscillations and the amplitude of the power envelope were similar between the two stimulation para- digms over sensorimotor cortices (Fig. 2), such that the relative phase relationship of the IAF/power envelope between the left and right hemisphere represents the major difference between the two stimulation protocols when analyzed within SM1. 

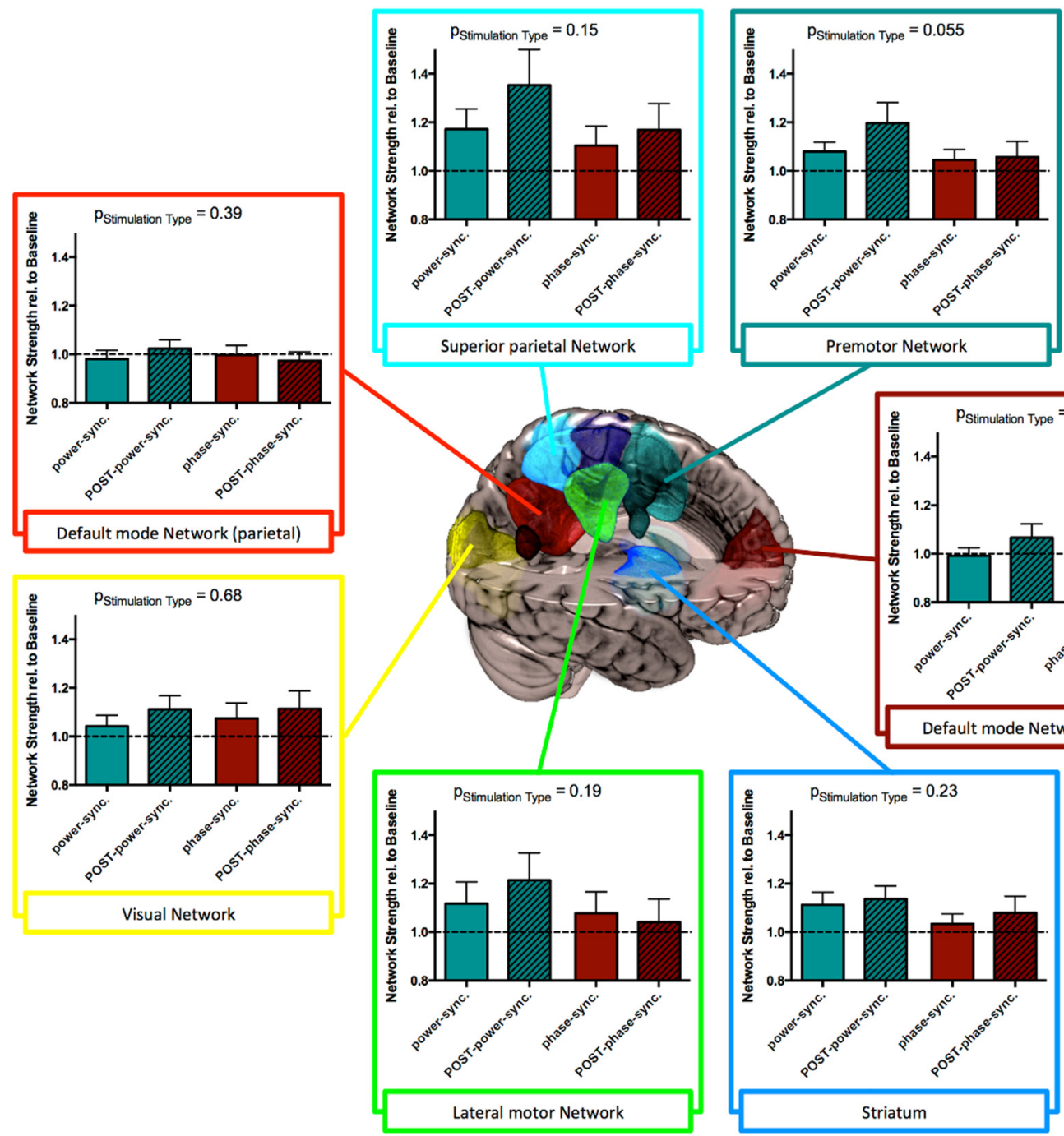

Figure 5. Results of other RSNs of interest. We did not find a main effect of stimulation type for any RSN other than the sensorimotor network. However, there was a similar trend (i.e., power-synchronized tACS increasing network connectivity vs phase-synchronized tACS not increasing network connectivity) observable in the striatum, the premotor network, the lateral motor network, and the superior parietal network. Although the effect did not reach statistical significance, the general trend fits the observed changes in the sensorimotor network because all of these networks are either associated with motor functions, and are therefore connected to the sensorimotor network (premotor network, striatum), or in between/under the stimulation electrodes (superior parietal network, lateral motor network). By contrast, we observed no difference between stimulation paradigms for the default-mode network (parietal and frontal) and the visual network, which rules out that the above described effects are purely driven by current injection itself or current related artifacts. $\mathrm{p}_{\text {stimulation Type }}$ iven for normalized data. Error bars indicate mean $\pm \mathrm{SEM}$.

However, the 3-electrode setup did not allow a perfect matching of the electric field distribution across the whole brain. In particular, the phase-synchronized stimulation also produces a strong electric field over occipital areas, which follows the carrier frequency but is no longer modulated by the envelope. Most importantly, the powersynchronized stimulation paradigm produces an in-phase fluctuation of the IAF envelope in the sensorimotor network, whereas phase-synchronized stimulation produces an antiphase fluctuation of the power envelope in the sensorimotor system.

\section{tACS effects on the sensorimotor RSN}

The same 20 subjects participated in a second session where we applied tACS using a 3-electrode setup (Fig. 1D), similar to Polanía et al. (2012) to the left and right SM1 inside an MR scanner to modulate rs-fMRI connectivity. In total, we acquired 5 restingstate scans for each subject (Fig. $1 D ; 7 \mathrm{~min}$, eyes-open, $3 \mathrm{~min}$ break between blocks). After measuring baseline connectivity (first run), either phase-synchronized or power-synchronized tACS was applied in the second and fourth run, respectively (or- der counterbalanced across subjects), and stimulation aftereffects were measured in runs 3 and 5 (POST phase-synchronized and POST power-synchronized). Subjects were aware that they would be stimulated, and 16 subjects reported feeling a slight tingling in the scanner, but they were not able to distinguish between the two stimulation regimens as established before the rs-fMRI scans via verbal report.

RSNs were spatially identified by group-ICA based on the whole dataset, and we further analyzed eight preselected RSNs of interest for further analysis (Fig. 3): the default-mode network (frontal and dorsal part, Fig. 3, red), a sensorimotor network (Fig. 3, dark blue), a premotor-network, the striatum (Fig. 3, blue-green), a lateral motor network (Fig. 3, green), a superior parietal network (Fig. 3, light blue), and a visual network (Fig. 3, yellow). We then compared the influence of the two tACS regimens on the different RSNs using a dual regression approach (Stagg et al., 2014). This approach yields a surrogate measure of synchronicity or coupling strength within the RSN under investigation. 


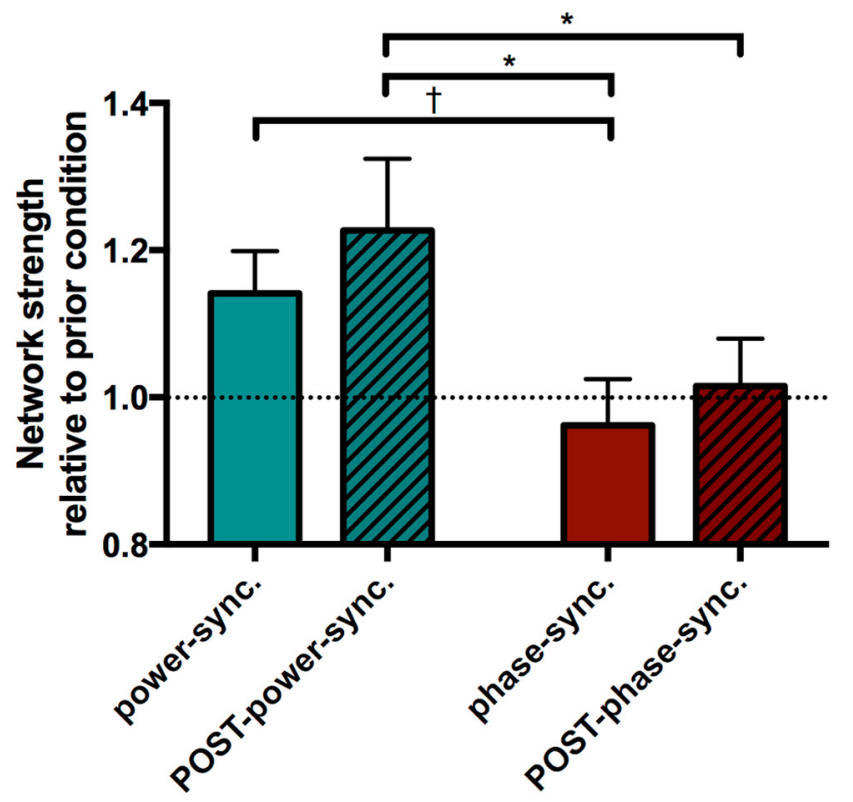

Figure 6. $\quad \mathrm{ACS}$ induced changes in network strength relative to the previous condition. Only power-synchronized stimulation signals cause a relative increase of connectivity strength, whereas phase-synchronized stimulation (i.e., power envelopes are in antiphase) has only a minor effect on connectivity. Error bars indicate mean \pm SEM. ${ }^{*} p<0.05 .{ }^{\dagger} p<0.1$.

Across all RSNs of interest, only the sensorimotor network changed its coupling strength significantly when tACS was applied (main effect of stimulation type $F_{(4,72)}=4.462, p=0.003$ in a linear mixed-effects model, including all five rs-fMRI runs). No other RSNs exhibited significant modulation effects (although the premotor network and the striatum approached significance, which is not surprising since these RSNs are strongly interconnected with motor areas; premotor network: $F_{(4,72)}=2.484, p=$ 0.051; striatum: $\left.F_{(4,72)}=2.102, p=0.089\right)$.

Next, we calculated a normalized index of network strength within the sensorimotor RSN to determine changes relative to the first baseline rs-fMRI scan (linear mixed effects model, 4 rs-fMRI runs normalized to baseline; Fig. 4). Again, we found a main effect of stimulation type only for the sensorimotor system $\left(F_{(3,57)}=4.402, p=0.007\right)$. Power-synchronized tACS increased connectivity strength by $25 \pm 9 \%$ (mean \pm SEM), which was significantly stronger than the effect of Phase-synchronized tACS ( $8 \pm 7 \%$ increase; $p=0.037$, Cohen's $d=0.49)$. Moreover, we found aftereffects of the power-synchronized tACS protocol on RSN connectivity (34 $\pm 8 \%$; compared with baseline) that were significantly larger than aftereffects $(12 \pm 6 \%, p=0.01$, Cohen's $d=0.4)$ or acute effects $(8 \pm 7 \%)$ caused by phasesynchronized tACS ( $p=0.002$, Cohens $d=0.62)$. Analogous results were revealed in a control analysis where the spatial topography of the RSNs was determined from an independent dataset, showing that the effect was robust to variations in RSN anatomy.

Importantly, after normalization to the baseline scan, the sensorimotor network was again the only network across all RSNs of interest that showed a significant main effect. Only the premotor network, but no longer the striatum, showed a trend toward significance (Fig. 5).

Because we observed aftereffects following both types of stimulation, we also calculated how tACS changed the network strength relative to the resting-state run immediately before stimulation (Fig. 6). For power-synchronized stimulation, we found similar increases during ( $14 \pm 5 \%$, mean \pm SEM) and after stim- ulation $(22 \pm 10 \%)$. For phase-synchronized tACS, we no longer observed an increase in connectivity during $(-4 \pm 6 \%)$ or after stimulation $(2 \pm 6 \%)$. Statistics still revealed a main effect of stimulation $\left(\operatorname{LMEM}, F_{(3,57)}=3.128, p=0.033\right)$ with significant post hoc differences between the aftereffects of the two stimulation types $(p=0.032)$ and a strong trend for the acute stimulation effects $(p=0.067)$.

We then analyzed order effects of the stimulation conditions; that is, we split the group of participants into those where the power-synchronized condition was applied first (i.e., following the baseline measurements) and those where the powersynchronized stimulation was applied second (i.e., on top of the aftereffects induced by the phase-synchronized stimulation) (Fig. 7 ). These results suggest that power-synchronized stimulation had a synchronizing effect regardless of order $(16 \pm 5 \%$ when applied as the first stimulation, and $12 \pm 6 \%$ when applied as the second stimulation). The phase-synchronized condition, however, only had a slight synchronizing effect when applied first $(5 \pm 7 \%)$, but a desynchronizing effect when applied second $(-13 \pm 7 \%)$.

Next, we performed a voxelwise analysis within the sensorimotor network to localize the effect previously observed at the level of the averaged network strength. Contrasting voxelwise RSN strength during power-synchronized tACS versus the baseline scan revealed a large cluster of voxels around the central sulcus, extending into the left middle cingulate cortex, left post central gyrus, and the right precentral and postcentral gyrus (Fig. 4 ; power-synchronized $>$ baseline, $\mathrm{p}_{\text {tfce-corr. }}<0.05$, thresholdfree cluster enhancement corrected). The size of this cluster increased even more when looking at aftereffects by comparing POST power-synchronized to baseline (paired $t$ test POST power-synchronized $>$ baseline, $\left.\mathrm{p}_{\text {tfce-corr. }}<0.05\right)$. By contrast, no voxel survived statistical thresholding when comparing phasesynchronized tACS or its aftereffects to baseline $\left(\mathrm{p}_{\text {tfce-corr. }}>0.05\right.$ for all voxels). The direct comparison of connectivity measured during power-synchronized versus phase-synchronized tACS revealed only two very small clusters close to the central sulcus in the left precentral and postcentral gyrus and the right postcentral gyrus (power-synchronized $>$ phase-synchronized, $\mathrm{p}_{\text {tfce-corr. }}<$ $0.05)$. According to our simulation, power-synchronized stimulation produces an electric field that peaks over the postcentral gyrus and stretches all along the central fissure from parietal to premotor regions (Fig. 2B). We would therefore expect the strongest effect for power-synchronized stimulation over postcentral regions, which is in line with the voxelwise analysis.

Our setup used a single return electrode over visual cortex that was the same size as the two stimulation electrodes. It is possible that this electrode placement might have induced subliminal phosphenes, especially during phase-synchronized stimulation, where the electric field is estimated to be almost twice as strong over occipital areas. Even though none of our subjects reported awareness of visual stimuli in the scanner when debriefed, possibly due to a relatively weak stimulation intensity (maximum amplitude $750 \mu \mathrm{A}$ ) in combination with the dimly lit scanner room, we performed a control analysis focused on the visual RSN. However, we found no statistical evidence that the visual RSN may have been modulated by either power-synchronized or phasesynchronized tACS stimulation (Fig. 5). This makes it very unlikely that the effects reported above were artifacts caused by visual flicker, a common side effect of tACS. This argument is further supported by the fact that the stronger rs-fMRI coupling within the sensorimotor RSN also persisted during the aftereffect runs (POST runs, without tACS stimulation). 
Even though the electrical field induced by phase-synchronized and powersynchronized stimulation was similar within the sensorimotor RSN (Fig. 2), it is not possible to match the induced electrical fields across the whole brain with a 3-electrode setup. In particular, the simulation revealed that phase-synchronized tACS affected parietal and occipital regions by entraining synchronous activity across hemispheres at the IAF. However, this did not result in higher RSN connectivity, suggesting that phase-synchronized stimulation was less effective in influencing rs-fMRI connectivity than powersynchronized stimulation. This was further confirmed by a control analysis of a parietal network (extending bilaterally from superior parietal cortex and middle occipital gyrus, and including a small cluster in right inferior frontal gyrus), which revealed no effect of stimulation type $\left(F_{(3,57)}=0.345, p=0.793\right)$.

Finally, we found no stimulation specific effect for the default-mode network (parietal and frontal), the superior parietal network or the lateral motor network, which rules out that the reported effects are purely driven by current injection itself or by artifacts possibly related to the current (Fig. 5). However, the superior parietal, premotor, and lateral motor network showed a similar response pattern as the sensorimotor network. We therefore ran an analysis on a full model (LMEM) incorporating all networks of interest and stimulation types. We found a significant main effect of stimulation type $\left(F_{(3,513)}=\right.$ 4.979, $p=0.002$ ) supporting our main claim that power-synchronized tACS is more effective for increasing rs-fMRI connectivity than phase-synchronized tACS. We also found a significant main effect of network $\left(F_{(6,513)}=5.176, p<0.001\right)$, but the stimulation type $\times$ network interaction was not significant $\left(F_{(18,513)}=\right.$ $0.646, p=0.863$ ), most likely because the electrical field spread widely (see Fig. $2 B$ ) and because intersubject variability was large. We also calculated Cohen's $d$ effect sizes between the powersynchronized versus phase-synchronized conditions for each of the networks and found a medium effect size only for the sensorimotor network (Cohen's $d=0.464)$. The next largest effect was in striatum (Cohen's $d=0.36$ ), whereas the effect sizes for all other networks were small (Cohen's $d=-0.152$ to 0.17 ). Thus, powersynchronized tACS had the strongest effect on the sensorimotor network, even though other networks were also mildly affected.

\section{Relationship between EEG connectivity and rs-fMRI connectivity within the sensorimotor RSN}

Next, we analyzed the effect of power-synchronized versus phasesynchronized tACS for each participant and found high interindividual variability (Fig. 8). To understand the possible origins of this variability, we investigated whether there is a relationship between interhemispheric connectivity measurements derived from the EEG data and the modulatory effect of the power-
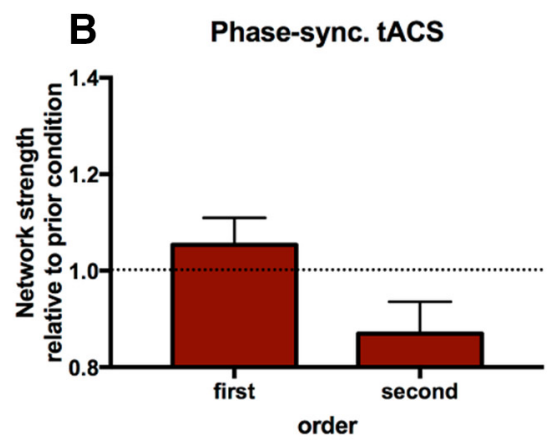

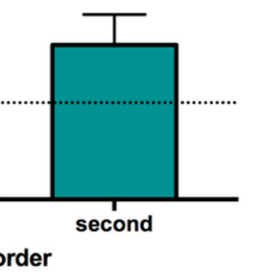

Figure 7. Effects of power-synchronized (left) and phase-synchronized stimulation (right) on network connectivity strength when participants were split depending on stimulation order. The data are normalized to the rest period immediately before stimulation. Power-synchronized stimulation has a synchronizing effect on the sensorimotor network regardless of whether it fol-

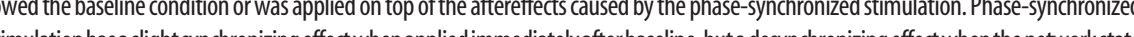
slight synchronizing effect when applied immediately after baseline, but a desynchronizing effe

A

Figure 8. Individual subject data. Left, Normalized network strength of the sensorimotor network during power-synchronized compared with phase-synchronized tACS. Right, Normalized network strength of the sensorimotor network during POST powersynchronized and POST phase-synchronized for each subject revealing large intersubject variability. Gray represents subjects who do not follow the general trend (power-synchronized tACS > phase-synchronized tACS).

synchronized or phase-synchronized tACS on rs-fMRI connectivity in the sensorimotor RSN. We found a negative correlation between the $\mathrm{r}_{\text {Env }}$ (Fig. $1 B$, blue) and the percentage increase in rs-fMRI connectivity strength within the sensorimotor network caused by power-synchronized tACS (Fig. 9A; Spearman's $\rho=$ $-0.481, p=0.032$ ). However, this correlation does not survive Bonferroni correction. Nonetheless, this indicates that subjects for whom the initial coupling of the IAF's power envelope was low tended to show a greater synchronization effect in response to power-synchronized stimulation. Moreover, we observed that those subjects who exhibited a strong natural interhemispheric coupling of IAF (i.e., high $\mathrm{r}_{\mathrm{IAF}}$ ) tended to respond stronger to power-synchronized tACS than those with a weak IAF coupling (Fig. $1 B$, red). Even though this association just failed to reach statistical significance (Fig. 9B; Spearman's $\rho=0.432, p=0.057$ ), it further suggests that rs-fMRI connectivity mostly reflects synchronous activity of the power envelopes.

No such correlations were found when $r_{\text {IAF }}$ and $r_{\text {Env }}$ were related to the percentage increase in rs-fMRI connectivity strength caused by phase-synchronized tACS (Fig. 9C,D).

Because phase-synchronized tACS could potentially influence $\alpha$ activity in occipital cortex, we ran control analyses to test whether 

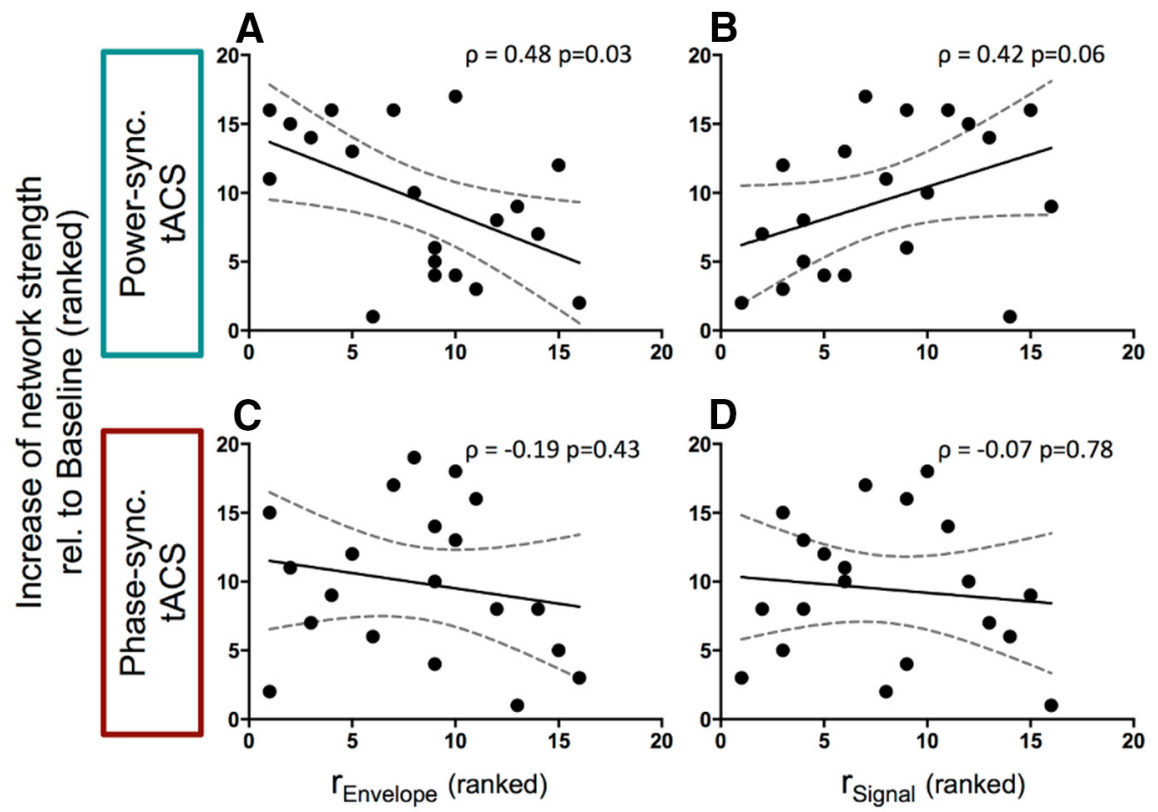

Figure 9. Correlations between $\mathrm{EEG}$ signal properties and percentage increase of network strength during power-synchronized and phase-synchronized tACS. $A$, Correlation between increase of network strength during power-synchronized tACS and IAF envelope correlation between electrode $C 3$ and $C 4\left(r_{\text {Envelope }}\right)$ revealing a negative association between the IAF envelope over sensorimotor cortex in the sensor space and efficacy of tACS stimulation $(\rho=-0.48, p=0.03) \cdot \boldsymbol{B}$, Positive correlation of increase of network strength during power-synchronized tACS and IAF signal correlation between electrode $C 3$ and $C 4\left(\mathrm{r}_{\text {signal }}\right)(\rho=0.42$, $p=0.06) . \boldsymbol{C}, \boldsymbol{D}$, No such relationship was visible between the change in network strength during phase-synchronized tACS and IAF envelope or signal between C3 and C4 (IAF envelope: $\rho=-0.19, p=0.43$; IAF signal: $\rho=-0.07, p=0.78$ ). EEG and fMRI measurements were performed in two separate sessions.

there was a correlation between increase in network strength and occipital $\alpha$ power or between increase in network strength and the difference between sensorimotor and occipital $\alpha$ frequency. Neither of these correlations was significant $(p \geq 0.12)$.

\section{Discussion}

The present results show that entraining the $\alpha$ power envelopes of remote yet connected areas in a synchronized fashion strengthens rs-fMRI connectivity within the targeted RSN. Increased connectivity strength was consistently observed regardless of whether the effect of power-synchronized tACS was compared with baseline or connectivity strength immediately before stimulation. This increase in connectivity outlasts the actual tACS stimulation period, suggesting some form of adaptation within the targeted neural circuits (Neuling et al., 2013; Vossen et al., 2015). Additionally, we demonstrated that the power-synchronized tACS regimen was more effective in modulating rs-fMRI connectivity when applied in individuals with weak electrophysiological interhemispheric coupling (i.e., less synchronized IAF power envelopes). Importantly, only small, nonsignificant effects were observed when the tACS signals phase-synchronized the IAF rhythm while the power envelopes oscillated in antiphase. Together, these findings provide causal evidence that power synchronization is an effective mechanism for linking oscillatory neural activity across different temporal scales. In particular, they demonstrate how the mechanism of cross-frequency coupling enables fast oscillatory rhythms to influence ultra-slow oscillations measured by interareal BOLD connectivity, an idea that was until now only supported by correlative evidence.

Finally, our results indicate that long-range connectivity can be modulated with tACS if the stimulation signals are appropriately designed. This might not only open new possibilities for experimental research but also lead to new therapeutic applications in diseases where resting-state connectivity is diminished as, for example, in autism or Alzheimer's disease (Greicius, 2008; Alaerts et al., 2014).

Here we used the $\alpha$ rhythm as a carrier frequency because it can be entrained by tACS (Zaehle et al., 2010; Neuling et al., 2013) and previous research revealed a systematic link between $\alpha$ power and the BOLD signal (Mantini et al., 2007; Ritter et al., 2009; Brookes et al., 2011; Hipp et al., 2012; Wang et al., 2012). However, power synchronization most likely represents a general mechanism that might be independent of the carrier frequency. Moreover, it is likely that rs-fMRI connectivity can also be modulated by directly synchronizing oscillations at $\leq 1 \mathrm{~Hz}$ (e.g., via optogenetic manipulations).

One might be surprised that applying phase-synchronized tACS, during which the power envelopes oscillated in antiphase, did not desynchronize the BOLD signal. However, synchronized IAF signals themselves might have a stabilizing effect if BOLD connectivity is relatively weak (Wang et al., 2012) as suggested by Figure 6, which shows that phase-synchronized tACS enhanced connectivity when the brain was in its typical connectivity state, as measured during the baseline scan (Fig. 7, right, condition first). However, if connectivity was enhanced, phase-synchronized tACS tended to reduce rs-fMRI connectivity (Fig. 7, right, condition second). By contrast, power-synchronized tACS consistently increased rs-fMRI connectivity measures regardless of the prior state (Fig. 7, left).

In addition to the acute effects of our stimulation paradigm, we also found significant increases in the resting-state run immediately after power-synchronized tACS.

A recent study reported changes in $\alpha$ power for up to $40 \mathrm{~min}$ after 10 min of tACS stimulation (Neuling et al., 2013). Currently, it is hypothesized that these prolonged tACS stimulation effects result from spike-timing-dependent plasticity (STDP), or from LTP/LTD (Vossen et al., 2015) causing an extended period of increased long-range connectivity as observed during the POST power-synchronized runs. Even though our study cannot speak to the underlying mechanism, it is promising from a therapeutic perspective that a relatively short stimulation period of 7 min causes longer-lasting aftereffects in modulating interhemispheric coupling strength. It would be interesting to test whether this aftereffect, which we measured at rest, would also be beneficial in the context of performing a task.

Our results suggest that there is an inverted relationship between the correlation of the EEG signal and the effect seen during power-synchronized tACS (i.e., low EEG correlation leads to a strong increase in rs-fMRI connectivity during powersynchronized tACS). No such relationship was observed with the phase-synchronized tACS condition. Two potential explanations for this observation are that entrainment is more efficient if the intrinsic interhemispheric connectivity of the IAF power enve- 
lopes is naturally weak, or that tACS entrainment does not yield additional benefits if the network is already highly synchronized.

\section{Distribution of the electric field}

One problem concerning noninvasive brain stimulation studies is the design of an appropriate control condition. Here we asked whether synchronizing $1 \mathrm{~Hz}$ oscillations across the left and right sensorimotor cortices via power-synchronized tACS increased connectivity within the sensorimotor network. We compared this with a control condition, which used the same principle tACS signals but the relative phase was shifted such that the IAF rhythms were synchronized while the power envelopes were in antiphase. Although the electric field strength is comparable between both tACS conditions for the sensorimotor network, the overall distribution of the electric field across the rest of the brain and the direction of the induced current differed. In particular, phase-synchronized stimulation produces an electric field shifted more toward occipital areas resulting in IAF stimulation that also had a strong effect on the primary visual cortex. Nevertheless, phase-synchronized tACS was rather ineffective for increasing connectivity within any of these RSNs, even though the modulation of IAF amplitude was relatively strong (up to $0.4 \mathrm{~V} / \mathrm{m}$ peak-to peak). This suggests that entrainment of rs-fMRI connectivity seems not to depend on the induced field strength alone, but also on the entrained frequency bands and their phase relationships. Our study suggests that the power-synchronized stimulation regimen is suitable for increasing rs-fMRI connectivity within RSNs, and it is possible that both the $0^{\circ}$ phase-shift of the power envelope and the $180^{\circ}$ phase-shift of the IAF are necessary for inducing this effect. In particular, it is likely that the carrier frequency (i.e., IAF) is necessary to entrain neuronal populations of cortex, but that the slow fluctuating envelope is necessary to synchronize fluctuations in the BOLD signal, which is measured by rs-fMRI connectivity metrics. This interpretation would explain why neither $1 \mathrm{~Hz}$ nor IAF stimulation alone was suitable for enhancing rs-fMRI (Vosskuhl et al., 2016). Moreover, it is in line with previous research suggesting that low-frequency components of the LFP (Lu et al., 2016) as well as low-frequency power envelopes of EEG/MEG oscillations are particularly strongly associated with BOLD fluctuations (Mantini et al., 2007; Brookes et al., 2011; Hipp et al., 2012).

In conclusion, most studies in humans relied on correlational approaches to investigate the relationship between fMRI measurements and electrophysiology because invasive electrical stimulation of the brain (i.e., direct electrical stimulation of cortex or deep brain stimulation) is usually restricted to clinical settings (Mandonnet et al., 2010; Bronstein et al., 2011). Compared with these methods, tACS has the advantage of being noninvasive and easy to apply. Here we showed that, if the waveforms are well designed, tACS is an appropriate tool for modulating the strength of RSNs. In particular, we provided causal evidence that power synchronization is effective for linking oscillatory neural activity across different temporal scales. This could open new opportunities for human fMRI research and new interventional approaches for modulating long-range connectivity of the human brain at rest. Potential therapeutic applications could be, for example, tACS-based modulation in patients with diminished resting-state connectivity within specific circuits, as for example in autism (Alaerts et al., 2014) and Alzheimer's disease (Greicius, 2008).

\section{References}

Alaerts K, Woolley DG, Steyaert J, Di Martino A, Swinnen SP, Wenderoth N (2014) Underconnectivity of the superior temporal sulcus predicts emo- tion recognition deficits in autism. Soc Cogn Affect Neurosci 9:15891600. CrossRef Medline

Alaerts K, Swinnen SP, Wenderoth N (2016) Sex differences in autism: a resting-state fMRI investigation of functional brain connectivity in males and females. Soc Cogn Affect Neurosci 11:1002-1016. CrossRef Medline

Antal A, Bikson M, Datta A, Lafon B, Dechent P, Parra LC, Paulus W (2014) Imaging artifacts induced by electrical stimulation during conventional fMRI of the brain. Neuroimage 85:1040-1047. CrossRef Medline

Beckmann CF, Mackay CE, Filippini N, Smith SM (2009) Group comparison of resting-state fMRI data using multi-subject ICA and dual regression. Neuroimage 47:S148. CrossRef

Berens P (2009) CircStat: a MATLABToolbox for circular statistics. J Statist Software 31:1-21.

Biswal B, Yetkin FZ, Haughton VM, Hyde JS (1995) Functional connectivity in the motor cortex of resting human brain using echo-planar MRI. Magn Reson Med 34:537-541. CrossRef Medline

Bronstein JM, Tagliati M, Alterman RL, Lozano AM, Volkmann J, Stefani A, Horak FB, Okun MS, Foote KD, Krack P, Pahwa R, Henderson JM, Hariz MI, Bakay RA, Rezai A, Marks WJ Jr, Moro E, Vitek JL, Weaver FM, Gross RE, et al. (2011) Deep brain stimulation for Parkinson disease: an expert consensus and review of key issues. Arch Neurol 68:165. CrossRef Medline

Brookes MJ, Woolrich M, Luckhoo H, Price D, Hale JR, Stephenson MC, Barnes GR, Smith SM, Morris PG (2011) Investigating the electrophysiological basis of resting state networks using magnetoencephalography. Proc Natl Acad Sci U S A 108:16783-16788. CrossRef Medline

Bullmore E, Sporns O (2009) Complex brain networks: graph theoretical analysis of structural and functional systems. Nat Rev Neurosci 10:186198. CrossRef Medline

Cecere R, Rees G, Romei V (2015) Individual differences in alpha frequency drive crossmodal illusory perception. Curr Biol 25:231-235. CrossRef Medline

Damoiseaux JS, Rombouts SA, Barkhof F, Scheltens P, Stam CJ, Smith SM, Beckmann CF (2006) Consistent resting-state networks across healthy subjects. Proc Natl Acad Sci U S A 103:13848-13853. CrossRef Medline

Delorme A, Makeig S (2004) EEGLAB: an open source toolbox for analysis of single-trial EEG dynamics including independent component analysis. J Neurosci Methods 134:9-21. CrossRef Medline

Fornito A, Bullmore ET (2010) What can spontaneous fluctuations of the blood oxygenation-level-dependent signal tell us about psychiatric disorders? Curr Opin Psychiatry 23:239-249. CrossRef Medline

Fox MD, Raichle ME (2007) Spontaneous fluctuations in brain activity observed with functional magnetic resonance imaging. Nat Rev Neurosci 8:700-711. CrossRef Medline

Greicius M (2008) Resting-state functional connectivity in neuropsychiatric disorders. Curr Opin Neurol 21:424-430. CrossRef Medline

Griffanti L, Salimi-Khorshidi G, Beckmann CF, Auerbach EJ, Douaud G, Sexton CE, Zsoldos E, Ebmeier KP, Filippini N, Mackay CE, Moeller S, Xu J, Yacoub E, Baselli G, Ugurbil K, Miller KL, Smith SM (2014) ICA-based artefact removal and accelerated fMRI acquisition for improved resting state network imaging. Neuroimage 95:232-247. CrossRef Medline

Haueisen J, Ramon C, Eiselt M, Brauer H, Nowak H (1997) Influence of tissue resistivities on neuromagnetic fields and electric potentials studied with a finite element model of the head. IEEE Trans Biomed Eng 44:727735. CrossRef Medline

He BJ, Snyder AZ, Zempel JM, Smyth MD, Raichle ME (2008) Electrophysiological correlates of the brain's intrinsic large-scale functional architecture. Proc Natl Acad Sci U S A 105:16039-16044. CrossRef Medline

Helfrich RF, Schneider TR, Rach S, Trautmann-Lengsfeld SA, Engel AK, Herrmann CS (2014) Entrainment of brain oscillations by transcranial alternating current stimulation. Curr Biol 24:333-339. CrossRef Medline

Herrmann CS, Rach S, Neuling T, Strüber D (2013) Transcranial alternating current stimulation: a review of the underlying mechanisms and modulation of cognitive processes. Front Hum Neurosci 7:279. CrossRef Medline

Hipp JF, Siegel M (2015) BOLD fMRI correlation reflects frequency-specific neuronal correlation. Curr Biol 25:1368-1374. CrossRef Medline

Hipp JF, Hawellek DJ, Corbetta M, Siegel M, Engel AK (2012) Large-scale cortical correlation structure of spontaneous oscillatory activity. Nat Neurosci 15:884-890. CrossRef Medline

Iacono MI, Neufeld E, Akinnagbe E, Bower K, Wolf J, Vogiatzis Oikonomidis I, Sharma D, Lloyd B, Wilm BJ, Wyss M, Pruessmann KP, Jakab A, Makris 
N, Cohen ED, Kuster N, Kainz W, Angelone LM (2015) MIDA: a multimodal imaging-based detailed anatomical model of the human head and neck. PLoS One 10:e0124126. CrossRef Medline

Liu Q, Balsters JH, Baechinger M, van der Groen O, Wenderoth N, Mantini D (2015) Estimating a neutral reference for electroencephalographic recordings: the importance of using a high-density montage and a realistic head model. J Neural Eng 12:056012. CrossRef Medline

Lu H, Zuo Y, Gu H, Waltz JA, Zhan W, Scholl CA, Rea W, Yang Y, Stein EA (2007) Synchronized $\delta$ oscillations correlate with the resting-state functional MRI signal. Proc Natl Acad Sci U S A 104:18265-18269. CrossRef Medline

Lu H, Wang L, Rea WW, Brynildsen JK, Jaime S, Zuo Y, Stein EA, Yang Y (2016) Low- but not high-frequency LFP correlates with spontaneous BOLD fluctuations in rat whisker barrel cortex. Cereb Cortex 26:683694. CrossRef Medline

Luck SJ (2014) An introduction to the event-related potential technique. Cambridge, MA: Massachusetts Institute of Technology.

Mandonnet E, Winkler PA, Duffau H (2010) Direct electrical stimulation as an input gate into brain functional networks: principles, advantages and limitations. Acta Neurochir 152:185-193. CrossRef Medline

Mantini D, Perrucci MG, Del Gratta C, Romani GL, Corbetta M (2007) Electrophysiological signatures of resting state networks in the human brain. Proc Natl Acad Sci U S A 104:13170-13175. CrossRef Medline

Moisa M, Polania R, Grueschow M, Ruff CC (2016) Brain network mechanisms underlying motor enhancement by transcranial entrainment of gamma oscillations. J Neurosci 36:12053-12065. CrossRef Medline

Neuling T, Rach S, Herrmann CS (2013) Orchestrating neuronal networks: sustained after-effects of transcranial alternating current stimulation depend upon brain states. Front Hum Neurosci 7:161. CrossRef Medline

Nir Y, Mukamel R, Dinstein I, Privman E, Harel M, Fisch L, Gelbard-Sagiv H, Kipervasser S, Andelman F, Neufeld MY, Kramer U, Arieli A, Fried I, Malach R (2008) Interhemispheric correlations of slow spontaneous neuronal fluctuations revealed in human sensory cortex. Nat Neurosci 11:1100-1108. CrossRef Medline

Oostenveld R, Fries P, Maris E, Schoffelen JM (2011) FieldTrip: open source software for advanced analysis of MEG, EEG, and invasive electrophysiological data. Comput Intell Neurosci 2011:1-9. CrossRef Medline

Pan WJ, Thompson GJ, Magnuson ME, Jaeger D, Keilholz S (2013) Infraslow LFP correlates to resting-state fMRI BOLD signals. Neuroimage 74:288-297. CrossRef Medline

Pascual-Marqui RD, Lehmann D, Koukkou M, Kochi K, Anderer P, Saletu B, Tanaka H, Hirata K, John ER, Prichep L, Biscay-Lirio R, Kinoshita T (2011) Assessing interactions in the brain with exact low-resolution electromagnetic tomography. Philos Trans A Math Phys Eng Sci 369:37683784. CrossRef Medline

Polanía R, Nitsche MA, Korman C, Batsikadze G, Paulus W (2012) The importance of timing in segregated theta phase-coupling for cognitive performance. Curr Biol 22:1314-1318. CrossRef Medline

Polanía R, Moisa M, Opitz A, Grueschow M, Ruff CC (2015) The precision of value-based choices depends causally on fronto-parietal phase coupling. Nat Commun 6:8090. CrossRef Medline

Ritter P, Moosmann M, Villringer A (2009) Rolandic alpha and beta EEG rhythms' strengths are inversely related to fMRI-BOLD signal in primary somatosensory and motor cortex. Hum Brain Mapp 30:1168-1187. CrossRef Medline

Salimi-Khorshidi G, Douaud G, Beckmann CF, Glasser MF, Griffanti L, Smith SM (2014) Automatic denoising of functional MRI data: combining independent component analysis and hierarchical fusion of classifiers. Neuroimage 90:449-468. CrossRef Medline

Schölvinck ML, Maier A, Ye FQ, Duyn JH, Leopold DA (2010) Neural basis of global resting-state fMRI activity. Proc Natl Acad Sci U S A 107: 10238-10243. CrossRef Medline

Siems M, Pape AA, Hipp JF, Siegel M (2016) Measuring the cortical correlation structure of spontaneous oscillatory activity with EEG and MEG. Neuroimage 129:345-355. CrossRef Medline

Stagg CJ, Bachtiar V, Amadi U, Gudberg CA, Ilie AS, Sampaio-Baptista C, O'Shea J, Woolrich M, Smith SM, Filippini N, Near J, Johansen-Berg H (2014) Local GABA concentration is related to network-level resting functional connectivity. eLife 3:e01465. CrossRef Medline

Thielscher A, Antunes A, Saturnino GB (2015) Field modeling for transcranial magnetic stimulation: a useful tool to understand the physiological effects of TMS? IEEE Eng Med Biol Soc 2015:222-225. CrossRef Medline

Vossen A, Gross J, Thut G (2015) Alpha power increase after transcranial alternating current stimulation at alpha frequency ( $\alpha$-tACS) reflects plastic changes rather than entrainment. Brain Stimul 8:499-508. CrossRef Medline

Vosskuhl J, Huster RJ, Herrmann CS (2016) BOLD signal effects of transcranial alternating current stimulation (tACS) in the alpha range: a concurrent tACS-fMRI study. Neuroimage 140:118-125. CrossRef Medline

Wang L, Saalmann YB, Pinsk MA, Arcaro MJ, Kastner S (2012) Electrophysiological low-frequency coherence and cross-frequency coupling contribute to BOLD connectivity. 76:1010-1020.

Winkler AM, Ridgway GR, Webster MA, Smith SM, Nichols TE (2014) Permutation inference for the general linear model. Neuroimage 92:381-397. CrossRef Medline

Woolley DG, Mantini D, Coxon JP, D’Hooge R, Swinnen SP, Wenderoth N (2015) Virtual water maze learning in human increases functional connectivity between posterior hippocampus and dorsal caudate. Hum Brain Mapp 36:1265-1277. CrossRef Medline

Zaehle T, Rach S, Herrmann CS (2010) Transcranial alternating current stimulation enhances individual alpha activity in human EEG. PLoS One 5:e13766. CrossRef Medline 